\title{
Two-dimensional conjugated polymers synthesized via on-surface chemistry
}

\author{
Chenguang $\mathrm{Li}^{1,2}$, Yongshuai Wang ${ }^{2,3}$, Huanli Dong ${ }^{2^{*}}$, Xiaotao Zhang ${ }^{1}$ and Wenping $\mathrm{Hu}^{1 *}$
}

Two-dimensional materials (2DMs) are an important subject in material science that have many interesting physical properties and significant potential applications in various technological areas [1,2]. Since the successful exfoliation of monoatomic graphene by Novoselov et al. [3] in 2004, a series of inorganic 2DMs, including transition-metal dichalcogenides (TMDs) [4], hexagonal boron nitride (h-BN) [5], metal nanomaterials [6], black phosphorus (BP) [7], and graphdiyne [8], have been obtained and investigated by fundamental studies in the fields of optoelectronics, catalysis, and energy storage [914]. Organic 2DMs, such as organic small molecules, polymers, and organic-inorganic hybrid materials, are emerging material systems currently attracting increasing attention owing to their unique advantages of a wide diversity of organic substrates, easily tunable properties/ functionalities, and diversified syntheses at low cost and high efficiency [15-23]. Among the 2DMs, 2D conjugated polymers (2DCPs) with long-range periodic covalently bonded structures and fully-extended conjugations have received special attention from scientists working in different fields. The tunable bandgap and diversified topological structures by appropriate molecular design [2428 ] endow 2DCPs, as a type of promising graphene analogue, with unique/tunable optoelectronic and spintronic properties, as well as promising applications in plastic optoelectronics and related functional devices/ circuits [29-35].

For the above mentioned fundamental studies and device applications, the most crucial issue of 2DCPs is their quality, which includes molecular orders, 2D-conjugated structures that determine the energy bandgaps/alignments and functionality of materials, and material sizes.
Thus far, the preparation of 2DCPs with the desired compositions, thicknesses, sizes, defects, crystal polymorphs, and surface properties is the core topic. Various synthetic processes based on both "top-down" and "bottom-up" strategies have been developed [2,17]. On-surface chemistry synthesis, a very important "bottom-up" strategy, was developed to overcome the limitations of uncontrollable polymer thickness, low yield, and low structural integrity [17,36,37]. On-surface chemistry, sometimes referred to as $2 \mathrm{D}$ chemistry, is the coupling of active molecules on a flat interface to directly provide ordered and expanded nanostructures to polymers (Fig. 1a). The interface provides a restricted system for the growth of precursors, which are capable of forming planar structures that cannot be accessed in a $3 \mathrm{D}$ reaction environment. Compared with classical solution-phase processes, another advantage of the surface method is the contribution of the related reaction conditions and potential surface interactions to the stabilization of highly active substances and intermediates. Moreover, on-surface chemistry synthesis is advantageous for directing the orientations of molecules to form ordered structures, and hence, it has been explored for the possibility of effectively controlling the morphologies and structures of 2DCPs. Therefore, an interfacial reaction has great potential in synthesizing substances that cannot be prepared by the traditional chemical method. At present, on-surface chemistry methods based on Ullmann coupling, Schiff-base condensation, Suzuki coupling, etc., to synthesize 2DCPs have been explored. For the preparation of 2DCPs, there are currently three kinds of interfaces: gassolid (mostly metal solid substrates with regular lattice structures), gas-liquid and liquid-liquid (Fig. 1b-d).

\footnotetext{
${ }^{1}$ Tianjin Key Laboratory of Molecular Optoelectronic Sciences, Department of Chemistry, School of Science, Tianjin University \& Collaborative Innovation Center of Chemical Science and Engineering, Tianjin 300072, China

${ }^{2}$ Beijing National Laboratory for Molecular Sciences, Key Laboratory of Organic Solids, Institute of Chemistry, Chinese Academy of Sciences, Beijing 100190, China

${ }^{3}$ University of Chinese Academy of Sciences, Beijing 100049, China

* Corresponding authors (emails: dhl522@iccas.ac.cn (Dong HL); huwp@tju.edu.cn (Hu WP))
} 


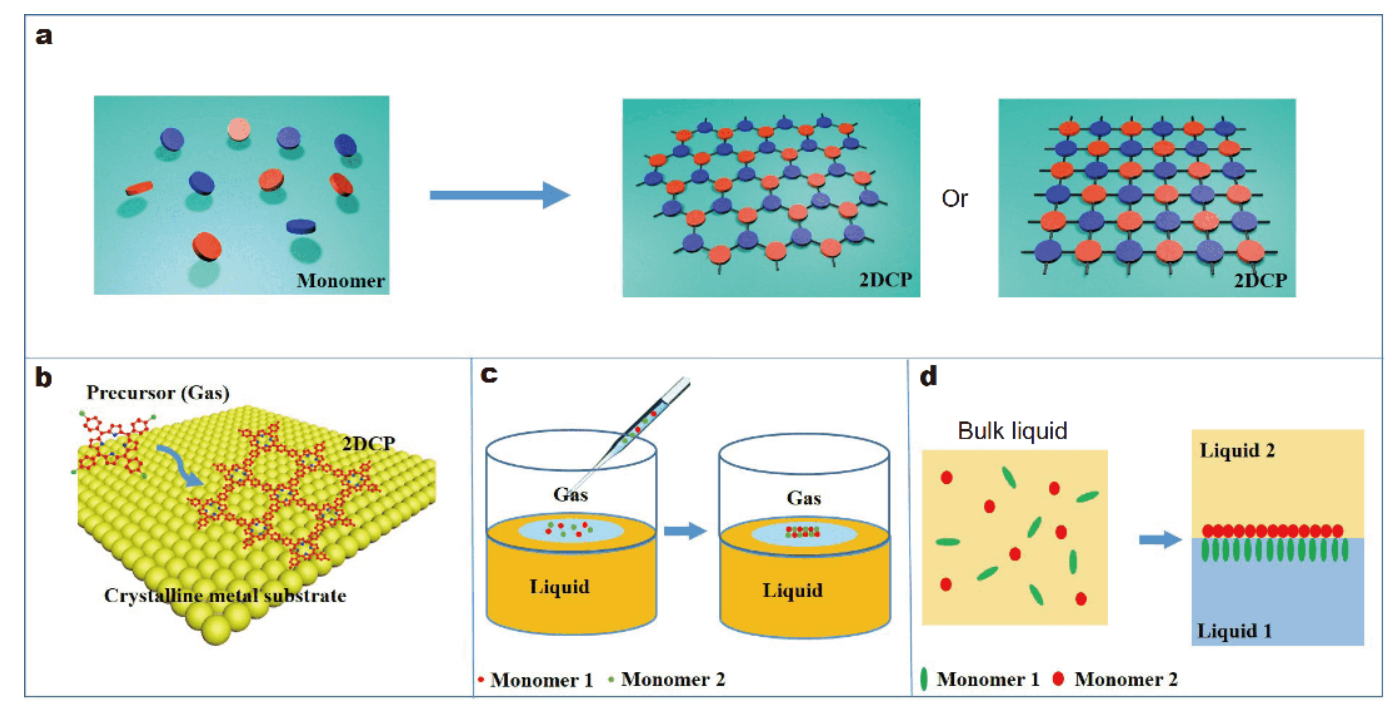

Figure 1 (a) Schematic illustrations of on-surface chemistry for synthesizing 2DCPs under conditions of (b) gas-solid, (c) gas-liquid, and (d) liquidliquid interface reactions.

The gas-solid interface without a solvent is the best means of studying the reaction process and creating more ordered structures. To analyze products directly at the molecular level, reactions are usually conducted directly in an ultra-high vacuum within the cavity of a scanning tunneling microscope (STM). Inspired by the pioneering investigations of the Ullmann coupling reaction via an on-surface chemistry setup by Hla et al. [38] in 2000, Grill et al. [39] formulated a strategy for the synthesis of covalently bound molecular nanostructures on a gold surface by the thermal annealing of active porphyrin molecules. This work paves the way for "bottom-up" synthetic stable 2D nano-architectures with possible future applications in molecular electronics. They also developed a sequential growth strategy to improve the quality of 2DCPs and made the construction of heterogeneous architecture highly selective (Fig. 2a) [40] by equipping a porphyrin building block with bromine and iodine side groups at orthogonal positions. Because of the different reactivities, the initial thermal activation of the iodine positions at low temperature resulted in a linear chain of porphyrin molecules and produced 2DCPs by the activation of the bromine positions through further heating. This strategy was able to maximize each molecule's adaptation to the optimal structure and avoidance of defect formations. Many other reactions have also been reported on gas-solid interfaces [41]. Although the metal interface synthesis strategy has made considerable progress, the fabrication of wafer-scale 2DCPs is still challenging, which is essential for basic research and device applications. The currently impressive and encouraging progress in the preparation of large-scale graphene, graphdiyne, and other 2D organic materials $[42,43]$ should stimulate research into large-area 2DCPs via appropriate experiment designs aiming at achieving high quality and efficient output at low cost.

In comparison with the gas-solid strategy, gas-liquid and liquid-liquid strategies are more robust because they do not require high-quality metal substrates with regular lattice structures, expensive equipment, or rigorous reaction conditions. Moreover, because of the fluidity of liquids, these strategies provide a flat and dynamic reaction environment favorable to efficient reactions and the preparation of large-scale 2D materials via facile modulation experimental conditions [44,45]. In 2016, Dai et al. [46] synthesized 2D covalent organic monolayers by the dynamic imine chemical method at the gas-liquid interface (Langmuir-Blodgett method) (Fig. 2b). Opening up new possibilities for the synthesis and structureproperty relationships of $2 \mathrm{DCPs}$, this attempt was the first time that the use of this interface obtained a monolayer sheet $(0.7 \mathrm{~nm}$ thickness) with lateral dimensions of tens of micrometers. Recently, Liu et al. [47] successfully synthesized a wafer-scale ultrathin $2 \mathrm{D}$ imine polymer film with controllable thickness from a simple benzene-1,3,5-tricarbaldehyde (BTA) and p-phenylenediamine (PDA) building block by using a Schiff-base polycondensation reaction at the air-water interface. Creating the potential for new applications, such 2D polymer films exhibited excellent switching performance 


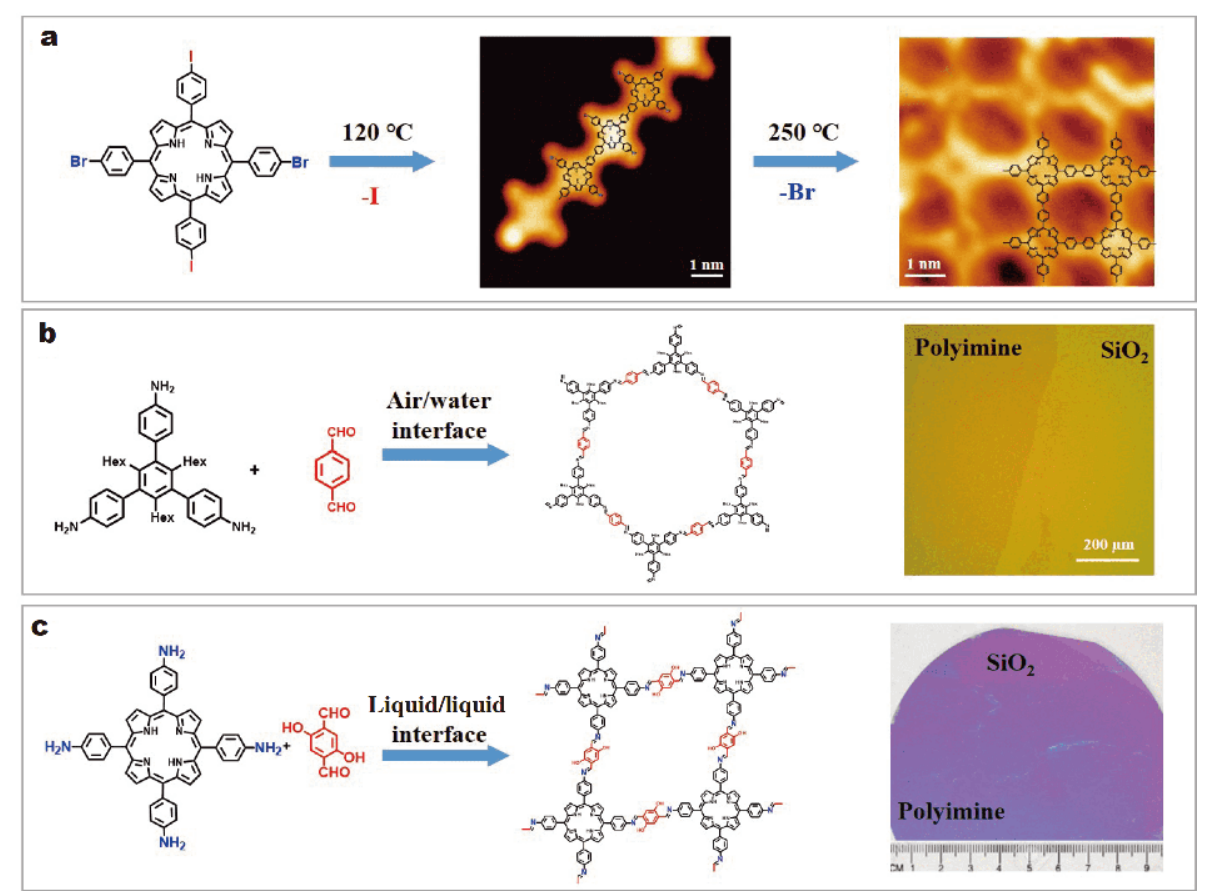

Figure 2 (a) Hierarchical growth following sequential thermal activation of 5,15-bis(4'-bromophenyl)-10,20-bis(4'-iodophenyl) porphyrin (trans$\mathrm{Br}_{2} \mathrm{I}_{2} \mathrm{TPP}$ ) building blocks on $\mathrm{Au}(111)$ substrate. Molecular structure of trans $-\mathrm{Br}_{2} \mathrm{I}_{2} \mathrm{TPP}$ (left), STM image of linear chains of porphyrin molecules after heating to $120^{\circ} \mathrm{C}$ with iodine dissociation. STM image of $2 \mathrm{D}$ polymer networks after heating the same sample to $250^{\circ} \mathrm{C}$ following bromine dissociation. Reproduced from Ref. [40]. Copyright 2012, Nature Publishing Group. (b) Synthesis of 2D polymine network from triamine and dialdehyde building blocks via LB method. Reproduced from Ref. [46]. Copyright 2016, John Wiley Sons, Inc. (c) Synthesis of wafer-sized multilayer of 2D polymer at water-chloroform interface. Reproduced from Ref. [50]. Copyright 2016, Nature Publishing Group.

with high reliability and reproducibility in memory devices because their intrinsically porous structures were conducive to the formation of conductive filaments during device operation. However, they have no proven crystallinity or highly ordered structures, which are crucial to the formation of efficient $\pi$-conjugation and high charge carrier transport in conjugated polymers [19,48,49]. In 2016, Sahabudeen et al. [50] disclosed the synthesis of crystalline 2DCPs based on porphyrin building blocks via Schiff-base condensation reactions at gas-liquid/liquid-liquid interfaces by reversible polycondensation (Fig. 2c). Under optimized experimental conditions, the sizes of the obtained polymers could be scaled up to the wafer scale. Moreover, semiconducting property was confirmed for the monolayer $2 \mathrm{D}$ polymer, which had a charge carrier mobility of $1.3 \times 10^{-6} \mathrm{~cm}^{2} \mathrm{~V}^{-1} \mathrm{~s}^{-1}$ in thin-film transistors without doping. More recently, Zhou et al. [51] further developed another simple and mild strategy to synthesize 2DCPs via Suzuki polymerization on a liquid-liquid interface. The products of the obtained porous graphene and porphyrincontaining polymers had large areas and were visible to the naked eye. For the porous graphene films, a carrier mobility of $3.2 \times 10^{-3} \mathrm{~cm}^{2} \mathrm{~V}^{-1} \mathrm{~s}^{-1}$ was examined and the catalytic activity of the hydrogen evolution reaction was detected.

The aforementioned examples are pioneering studies in this emerging field of on-surface chemistry. Although the syntheses of some multilayer 2DCPs with controllable sizes and thicknesses at the atomic or molecular level have been realized by the interface synthesis strategy, many problems remain to be solved. The first problem is controlling the growth of 2DCPs to obtain high-quality products with definite crystalline phases, grain sizes, and grain boundaries. Also, the design of more convenient transfer methods is required because of the relatively low mechanical stability of 2DCPs. Finally, the applications of 2DCPs in optoelectronic devices are rare, while the resulting performances, such as charge and exciton transport, are still very low $[29,30,50-52]$, and, in fact, are far from the expected values, which are highly dependent on controlling the structures of the 2DCPs at the atomic and molecular levels. Thus, increased efforts and the search for efficient reactions occurring at the surface for highly ordered structures at molecular level and desired energy band structure by molecular design are required in this 
field. It is believed that more amazing results will be achieved in the future under the joint efforts of scientists from the fields of chemical sciences, material sciences, and device physics.

Received 6 July 2019; accepted 30 July 2019; published online 28 August 2019

1 Geim AK, Novoselov KS. The rise of graphene. Nat Mater, 2007, 6: 183-191

2 Tan C, Cao X, Wu XJ, et al. Recent advances in ultrathin twodimensional nanomaterials. Chem Rev, 2017, 117: 6225-6331

3 Novoselov KS, Geim AK, Morozov SV, et al. Electric field effect in atomically thin carbon films. Science, 2004, 306: 666-669

4 Lv R, Robinson JA, Schaak RE, et al. Transition metal dichalcogenides and beyond: Synthesis, properties, and applications of single- and few-layer nanosheets. Acc Chem Res, 2015, 48: 56-64

5 Weng Q, Wang X, Wang X, et al. Functionalized hexagonal boron nitride nanomaterials: Emerging properties and applications. Chem Soc Rev, 2016, 45: 3989-4012

6 Fan Z, Huang X, Tan C, et al. Thin metal nanostructures: Synthesis, properties and applications. Chem Sci, 2015, 6: 95-111

7 Liu H, Du Y, Deng Y, et al. Semiconducting black phosphorus: Synthesis, transport properties and electronic applications. Chem Soc Rev, 2015, 44: 2732-2743

8 Li G, Li Y, Liu H, et al. Architecture of graphdiyne nanoscale films. Chem Commun, 2010, 46: 3256-3258

9 Jin $\mathrm{H}$, Guo C, Liu X, et al. Emerging two-dimensional nanomaterials for electrocatalysis. Chem Rev, 2018, 118: 6337-6408

10 Das S, Pandey D, Thomas J, et al. The role of graphene and other 2D materials in solar photovoltaics. Adv Mater, 2018, 31: 1802722

$11 \mathrm{Hu}$ W, Yang J. Two-dimensional van der Waals heterojunctions for functional materials and devices. J Mater Chem C, 2017, 5: 12289-12297

12 Wu G, Huang J, Zang Y, et al. Porous field-effect transistors based on a semiconductive metal-organic framework. J Am Chem Soc, 2017, 139: 1360-1363

13 Lin $\mathrm{H}$, Chen $\mathrm{L}, \mathrm{Lu} \mathrm{X}$, et al. Two-dimensional titanium carbide MXenes as efficient non-noble metal electrocatalysts for oxygen reduction reaction. Sci China Mater, 2019, 62: 662-670

14 Gong X, Liu Y, Xiang H, et al. Membraneless reproducible $\mathrm{MoS}_{2}$ field-effect transistor biosensor for high sensitive and selective detection of FGF21. Sci China Mater, 2019, 62: 1479-1487

15 Yang F, Cheng S, Zhang X, et al. 2D organic materials for optoelectronic applications. Adv Mater, 2018, 30: 1702415

16 Cao M, Zhang C, Cai Z, et al. Enhanced photoelectrical response of thermodynamically epitaxial organic crystals at the two-dimensional limit. Nat Commun, 2019, 10: 756

17 Dong R, Zhang T, Feng X. Interface-assisted synthesis of 2D materials: trend and challenges. Chem Rev, 2018, 118: 6189-6235

$18 \mathrm{Fu} \mathrm{Q}, \mathrm{Bao} \mathrm{X}$. Surface chemistry and catalysis confined under twodimensional materials. Chem Soc Rev, 2017, 46: 1842-1874

19 Zhang Z, Liu Q, Dong H, et al. Conjugated polymer crystals via topochemical polymerization. Sci China Chem, 2019, doi: 10.1007/ s11426-019-9495-4

20 Zhan X, Chen Z, Zhang Q. Recent progress in two-dimensional COFs for energy-related applications. J Mater Chem A, 2017, 5: 14463-14479

21 Zhang W, Zhang L, Zhao H, et al. A two-dimensional cationic covalent organic framework membrane for selective molecular sieving. J Mater Chem A, 2018, 6: 13331-13339

22 Xu SQ, Zhan TG, Wen Q, et al. Diversity of covalent organic frameworks (COFs): A 2D COF containing two kinds of triangular micropores of different sizes. ACS Macro Lett, 2016, 5: 99-102

23 Shinde DB, Sheng G, Li X, et al. Crystalline 2D covalent organic framework membranes for high-flux organic solvent nanofiltration. J Am Chem Soc, 2018, 140: 14342-14349

24 Feng X, Schlüter AD. Towards macroscopic crystalline 2D polymers. Angew Chem Int Ed, 2018, 57: 13748-13763

25 Zhang Q, Dong H, Hu W. Electrochemical polymerization for twodimensional conjugated polymers. J Mater Chem C, 2018, 6: 10672-10686

26 Huang N, Wang P, Jiang D. Covalent organic frameworks: A materials platform for structural and functional designs. Nat Rev Mater, 2016, 1: 19

27 Liu W, Loh KP. Two-dimensional conjugated polymers based on C-C coupling. Acc Chem Res, 2017, 50: 522-526

28 Cardenas L, Gutzler R, Lipton-Duffin J, et al. Synthesis and electronic structure of a two dimensional $\pi$-conjugated polythiophene. Chem Sci, 2013, 4: 3263-3268

29 Liu J, Zan W, Li K, et al. Solution synthesis of semiconducting twodimensional polymer via trimerization of carbonitrile. J Am Chem Soc, 2017, 139: 11666-11669

30 Feldblyum JI, McCreery CH, Andrews SC, et al. Few-layer, largearea, 2D covalent organic framework semiconductor thin films. Chem Commun, 2015, 51: 13894-13897

31 Jin W, Liu L, Yang T, et al. Exploring Peltier effect in organic thermoelectric films. Nat Commun, 2018, 9: 3586

32 Jin E, Asada $\mathrm{M}, \mathrm{Xu} \mathrm{Q}$, et al. Two-dimensional sp ${ }^{2}$ carbon-conjugated covalent organic frameworks. Science, 2017, 357: 673-676

33 Liu J, Lyu P, Zhang Y, et al. New layered triazine framework/ exfoliated $2 \mathrm{D}$ polymer with superior sodium-storage properties. Adv Mater, 2018, 30: 1705401

34 Huang X, Sheng P, Tu Z, et al. A two-dimensional $\pi-\mathrm{d}$ conjugated coordination polymer with extremely high electrical conductivity and ambipolar transport behaviour. Nat Commun, 2015, 6: 7408

35 Lin ZQ, Xie J, Zhang BW, et al. Solution-processed nitrogen-rich graphene-like holey conjugated polymer for efficient lithium ion storage. Nano Energy, 2017, 41: 117-127

36 Liu W, Luo X, Bao Y, et al. A two-dimensional conjugated aromatic polymer via C-C coupling reaction. Nat Chem, 2017, 9: 563570

37 Duan H, Lyu P, Liu J, et al. Semiconducting crystalline two-dimensional polyimide nanosheets with superior sodium storage properties. ACS Nano, 2019, 13: acsnano.8b09416

38 Hla SW, Bartels L, Meyer G, et al. Inducing all steps of a chemical reaction with the scanning tunneling microscope tip: Towards single molecule engineering. Phys Rev Lett, 2000, 85: 2777-2780

39 Grill L, Dyer M, Lafferentz L, et al. Nano-architectures by covalent assembly of molecular building blocks. Nat Nanotech, 2007, 2: 687-691

40 Lafferentz L, Eberhardt V, Dri C, et al. Controlling on-surface polymerization by hierarchical and substrate-directed growth. Nat Chem, 2012, 4: 215-220

41 Held PA, Fuchs H, Studer A. Covalent-bond formation via onsurface chemistry. Chem Eur J, 2017, 23: 5874-5892

42 Jiang $\mathrm{L}, \mathrm{Niu} \mathrm{T}, \mathrm{Lu} \mathrm{X}$, et al. Low-temperature, bottom-up synthesis of graphene via a radical-coupling reaction. J Am Chem Soc, 2013, 135: $9050-9054$ 
43 Liu R, Gao X, Zhou J, et al. Chemical vapor deposition growth of linked carbon monolayers with acetylenic scaffoldings on silver foil. Adv Mater, 2017, 29: 1604665

$44 \mathrm{Xu} \mathrm{C}$, He P, Liu J, et al. A general method for growing twodimensional crystals of organic semiconductors by "solution epitaxy". Angew Chem Int Ed, 2016, 55: 9519-9523

45 Wang Q, Yang F, Zhang Y, et al. Space-confined strategy toward large-area two-dimensional single crystals of molecular materials. J Am Chem Soc, 2018, 140: 5339-5342

46 Dai W, Shao F, Szczerbiński J, et al. Synthesis of a two-dimensional covalent organic monolayer through dynamic imine chemistry at the air/water interface. Angew Chem Int Ed, 2016, 55: 213-217

47 Liu J, Yang F, Cao L, et al. A robust nonvolatile resistive memory device based on a freestanding ultrathin 2D imine polymer film. Adv Mater, 2019, 31: 1902264

48 Yao Y, Dong H, Liu F, et al. Approaching intra- and interchain charge transport of conjugated polymers facilely by topochemical polymerized single crystals. Adv Mater, 2017, 29: 1701251

49 Dong HL, Yan QQ, Hu WP, et al. Multilevel investigation of charge transport in conjugated polymers-new opportunities in polymer electronics. Acta Polym Sin, 2017, 8: 1246-1260

50 Sahabudeen H, Qi H, Glatz BA, et al. Wafer-sized multifunctional polyimine-based two-dimensional conjugated polymers with high mechanical stiffness. Nat Commun, 2016, 7: 13461

51 Zhou D, Tan X, Wu H, et al. Synthesis of C-C bonded twodimensional conjugated covalent organic framework films by suzuki polymerization on a liquid-liquid interface. Angew Chem Int Ed, 2019, 58: 1376-1381

52 Mahmood J, Lee EK, Jung M, et al. Nitrogenated holey two-dimensional structures. Nat Commun, 2015, 6: 6486

Acknowledgements This work was supported by the National Natural Science Foundation of China (51725304, 51633006, 21875259, 91833306, 61890943 and 21661132006), the Ministry of Science and Technology of China (2017YFA0204503 and 2016YFB0401100), the Strategic Priority Research Program (XDB12000000) of the Chinese Academy of Sciences, the Youth Innovation Promotion Association of the Chinese Academy of Sciences, and the National Program for Support of Top-notch Young Professionals.

Author contributions Dong $\mathrm{H}$ and $\mathrm{Hu} \mathrm{W}$ designed the project. Li C and Dong $\mathrm{H}$ co-wrote the manuscript. All authors contributed to the general discussion.

Conflict of interest The authors declare no conflict of interest.

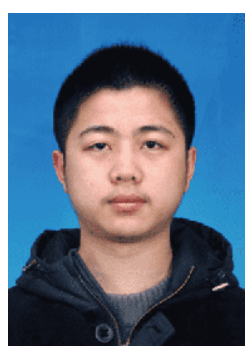

Chenguang $\mathbf{L i}$ is a $\mathrm{PhD}$ candidate (supervisor: Prof. Wenping $\mathrm{Hu}$ ) in the Department of Chemistry, School of Science, Tianjin University. He received his Bachelor's degree (2014) from Tianjin University and Master's degree (2017) from Lanzhou University. His interest focuses on the design, characterization, and optoelectronic properties of $2 \mathrm{D}$ conjugated polymers.

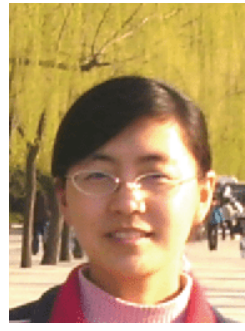

Huanli Dong is a Professor of the Institute of Chemistry, Chinese Academy of Sciences (ICCAS). She received her $\mathrm{PhD}$ from the Institute in 2009 after she got her MSc from Fujian Institute of Research on the Structure of Material, CAS, in 2006. Her current research focuses on the organic semiconductor crystals, including organic small molecules, and conjugated polymers by material science and growth technology, as well as their applications in OFETs, integrated optoelectronic devices, and circuits.

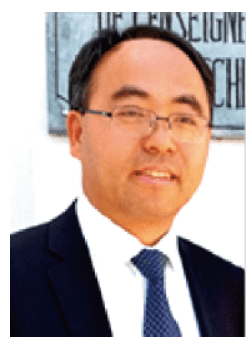

Wenping Hu is a Professor of Tianjin University and a Cheung Kong Professorship of the Ministry of Education, China. He received his $\mathrm{PhD}$ from ICCAS in 1999. Then, he joined Osaka University and Stuttgart University as a research fellow of Japan Society for the Promotion of Sciences and an Alexander von Humboldt fellow, respectively. In 2003, he worked with Nippon Telephone and Telegraph, and then joined ICCAS as a full professor. He moved to Tianjin University in 2013 and was promoted to vice president of the university in 2016. His research interest focuses on organic optoelectronics.

\section{界面合成二维共轭聚合物}

李晨光 $^{1,2}$, 王永帅 ${ }^{2,3}$, 董焕 丽 $^{2 *}$, 张小涛, 胡文平 ${ }^{1 *}$

摘要 二维共轭聚合物具有平面共轭结构和分子中离域的 $\pi$ 电子体 系, 因而具有很强的平面内电荷传输能力, 表现出独特的物理和化 学性质, 在光学、电学、传感等领域具有广阔的应用前景. 但如何 获得分子结构及形貌符合二维特征、且尺寸满足制作电子器件需 求的分子非常困难, 是广大科研人员急需解决的问题. 本文总结了 界面合成二维共轭聚合物的方法, 讨论了这个领域目前面临的问 题和挑战, 并指出了未来的研究方向. 